\title{
Evidence of carrier leakage into the L-valley in InAs-based quantum cascade lasers under high hydrostatic pressure
}

\author{
Igor P. Marko*, , Alfred R. Adams', Stephen J. Sweeney', Roland Teissier², Alexei N. Baranov², \\ and Stanko Tomic ${ }^{3}$ \\ ${ }^{1}$ Advanced Technology Institute, University of Surrey, Guildford, Surrey, GU2 7XH, UK \\ ${ }^{2}$ Institut d'Électronique du Sud, UMR5214, CNRS/Université Montpellier 2, 34095 Montpellier, France \\ ${ }^{3}$ Computational Science and Engineering Department, STFC Daresbury Laboratory, Cheshire WA4 4AD, UK
}

Received 16 June 2008, accepted 28 August 2008

Published online 8 December 2008

PACS 42.55.Px, 62.50.-p, 72.10.-d, 73.21.Fg, 73.63.Hs, 85.35.Be

* Corresponding author: e-mail i.marko@surrey.ac.uk, Phone: +44 (0)1483 689 409, Fax: +44 (0)1483 689404

To investigate carrier scattering processes in short wavelength InAs/AlSb quantum cascade lasers we carried out experimental and theoretical studies of the threshold current, $I_{\mathrm{th}}$, as a function of high hydrostatic pressure and temperature. Using the calculated pressure dependence of the optical phonon scattering current, $I_{\mathrm{ph}}$, and the estimated pressure dependence of leakage current, $I_{\text {leak }}$, we show that carrier leakage from the upper laser levels into the indirect L-valley of the conduction band in InAs quantum wells is negligible in the 3.3 $\mu \mathrm{m}$ QCLs at RT leading to their superior temperature performance. In the shorter wavelength devices emitting at $2.9 \mu \mathrm{m}$, this loss mechanism is more important and accounts for up to $13 \%$ of $I_{\text {th }}$ at $190 \mathrm{~K}$.

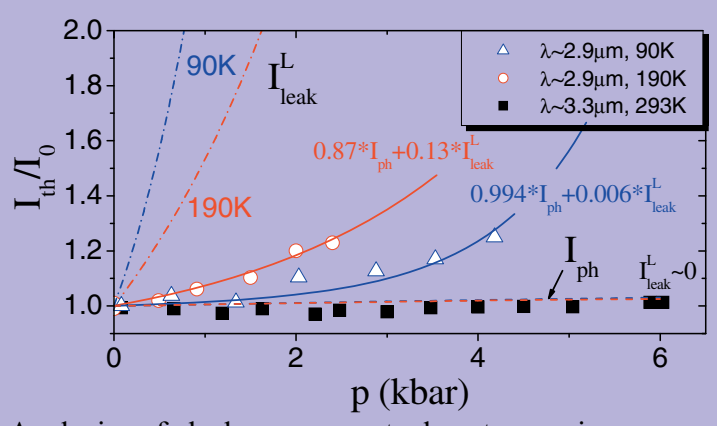

Analysis of leakage current due to carrier escape into L-minimum of conduction band under high hydrostatic pressure.
1 Introduction Developing lasers for the midinfrared spectral region $3-4 \mu \mathrm{m}$ is of key importance for gas sensing and free space optical communications. There are several strategies being investigated to cover this spectral range using inter-band quantum well or cascade lasers. However the performance of such lasers tends to deteriorate with increasing emission wavelength due to increasing non-radiative Auger recombination and optical losses. Quantum Cascade Lasers (QCLs) have been very successful above $4 \mu \mathrm{m}[1,2]$ and considerable effort is now being put into reducing the wavelength of QCLs to target the shorter wavelength applications [3, 4]. Potential advantages of QCLs are high temperature stability of the threshold current, $I_{\mathrm{th}}$, (characterised by high $T_{0}$ values: $\left.T_{0}=I_{\mathrm{th}}\left(\mathrm{d} I_{\mathrm{th}} / \mathrm{d} T\right)^{-1}\right)$ and high output power. Here we discuss short wavelength InAs/AlSb quantum cascade lasers emitting around $3 \mu \mathrm{m}[4]$.

It is anticipated that QCLs will overcome the problem of non-radiative Auger recombination which plagues interband lasers. Modelling, however, shows that the operation of short wavelength QCLs may be affected by electron leakage from the upper levels of the laser transition into energy states associated with indirect conduction band valleys. Compared with other materials used at present in QCLs the InAs/AlSb system exhibits not only the highest conduction band offset $(2.1 \mathrm{eV})$, but also the largest separation of direct $(\Gamma)$ and indirect $(\mathrm{L})$ minima in the quantum well conduction band $(0.72-0.76 \mathrm{eV})$ [3]. To investigate the presence and importance of the L-leakage loss mechanism, we analysed the pressure dependences of the lasing 
threshold current, $I_{\text {th }}$, in InAs/AlSb QCLs as a function of temperature. High hydrostatic pressure enables one to tune the separation between the $\Gamma$ and L minima of the conduction band at constant temperature providing an effective tool to separate and study the effects related to band structure and temperature.

2 Experiment QCLs emitting near $2.9 \mu \mathrm{m}$ and $3.3 \mu \mathrm{m}$ were grown by $\mathrm{MBE}$ and processed into ridge lasers (ridge widths of $12 \mu \mathrm{m}$ and $17 \mu \mathrm{m}$ ) using wet chemical etching and standard photolithography. Lasers were cleaved in cavities with lengths of 1.1-2.05 mm. Details of the growth and structure of the lasers can be found in $[3,4]$.

The lasers were investigated in the temperature range of $80-295 \mathrm{~K}$ using a static gas exchange cryostat. Lasers were operated in pulsed mode with a repetition rate of $1 \mathrm{kHz}$ and pulse width of $100 \mathrm{~ns}$. The emission intensity was measured using a cooled InSb detector and lock-in amplifier. For the high pressure studies the lasers were mounted in the pressure cell shown in Fig. 1, which was then placed in a cryostat cooled with liquid nitrogen. The cell was connected to a helium gas compressor, which can generate pressures up to $10 \mathrm{kbar}(1 \mathrm{GPa})$.

3 Results and discussion Figure 2 shows the temperature dependence of the threshold current density for both of the QCLs. The laser emitting at $2.9 \mu \mathrm{m}$ exhibited a higher threshold current and lower maximum operation temperature, $T_{\max }$, compared with the longer wavelength device. The threshold current density of a QCL at its maximum operation temperature corresponds to it entering into the negative differential resistance (NDR) region of the current-voltage characteristics which indicates misalignment of energy levels in the active region at high elec-

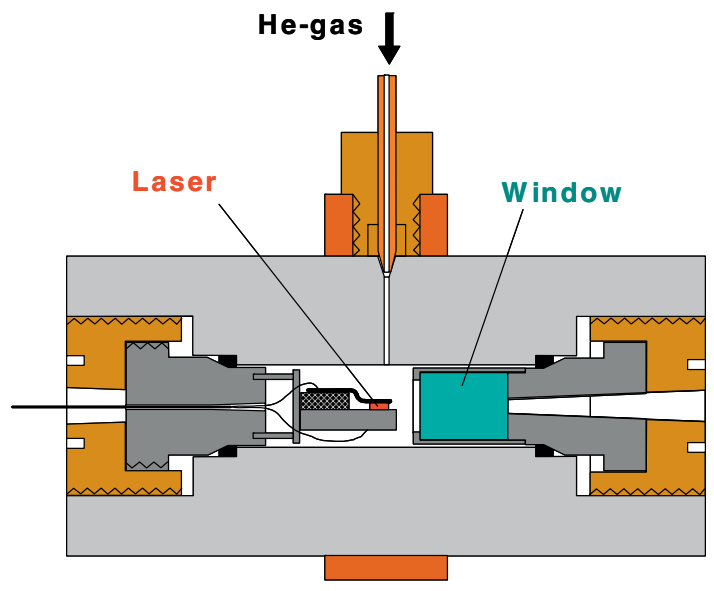

Figure 1 (online colour at: www.pss-b.com) High pressure cell used in the experiments. Lasers were mounted in a clip and the facet emission was collected from a sapphire optical window. The pressure cell was mounted in a cryostat for measurements at different temperatures.

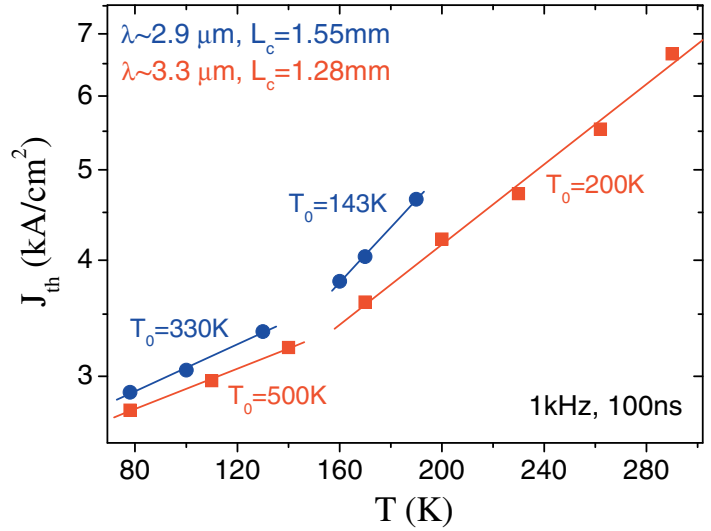

Figure 2 (online colour at: www.pss-b.com) Temperature dependence of threshold current density of the two InAs/AlSb based QCLs with emission wavelengths near $2.9 \mu \mathrm{m}$ and $3.3 \mu \mathrm{m}$.

tric field resulting in switching the laser action off. It should be noted that by using longer cavities $(\sim 4 \mathrm{~mm})$ it was possible to reach a high $T_{\max }$ of $280 \mathrm{~K}$ and $400 \mathrm{~K}$ for the $2.9 \mu \mathrm{m}$ and $3.3 \mu \mathrm{m}$ devices, respectively $[3,4]$. The difference in $T_{\max }$ may indicate that a temperature sensitive loss process in these lasers is more important at shorter wavelengths, which is in agreement with the assumption of indirect carrier leakage into the $\mathrm{L}$ minimum of the conduction band, since the shorter wavelength devices have a smaller $\Gamma-\mathrm{L}$ separation.

High hydrostatic pressure results provide further evidence that carrier scattering between the $\Gamma$ and $\mathrm{L}$ valleys plays a significant role in operation of short-wavelength QCLs. Figure 3 shows the pressure dependence of $I_{\text {th }}$ at different temperatures for two such QC lasers with different lasing wavelengths. We assume that the total threshold current is formed by two components: current due to intersubband longitudinal optical phonon scattering, $I_{\mathrm{ph}}$, and current loss due to the leakage into the L-valleys, $I_{\text {leak }}^{\mathrm{L}}$ (the current component related to radiative transitions is very small in QCLs at threshold and may be neglected in this analysis). The dashed lines $\left(I_{\mathrm{ph}}\right)$ in Fig. 3 describe the pressure dependence of inter-subband carrier relaxation via longitudinal polar optical phonon scattering calculated using the Frölich Hamiltonian approximation [5].

The theoretical pressure variation of the leakage current is also plotted in the figure for different temperatures and was derived using the temperature dependence of the leakage current at threshold which may be calculated from Eq. (1) [6]:

$$
I_{\text {leak }}^{\mathrm{L}}(T)=I_{0 \text { leak }} \exp \left(-\frac{E_{\mathrm{a}}}{k T}\right),
$$

where $I_{\text {0leak }}$ is a constant, $k$ is the Boltzmann constant, and $E_{\mathrm{a}}$ is the activation energy of the leakage process, which is equal to the energy separation between the quasi-Fermi level (assumed to be at the upper lasing level) and L-related 


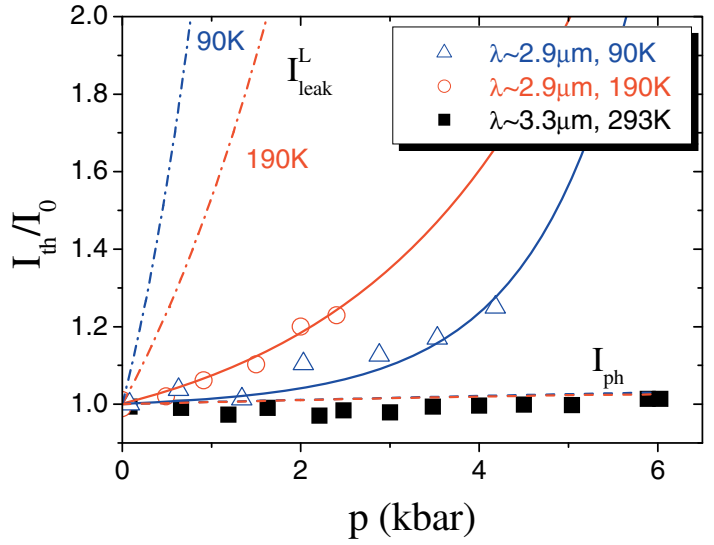

Figure 3 (online colour at: www.pss-b.com) Symbols show experimental dependencies of the normalised threshold current to its value at atmospheric pressure, $I_{0}$, measured for the two QCLs at different temperatures $(2.9 \mu \mathrm{m}$ device with cavity length of $2.05 \mathrm{~mm}$ and $3.3 \mu \mathrm{m}$ device with cavity length of $1.28 \mathrm{~mm}$ ). The dashed lines present theoretical calculations of the pressure dependence of intersubband carrier relaxation via longitudinal polar optical phonon scattering in the shorter wavelength device. Dashdotted lines show the current due to the carrier leakage into the $\mathrm{L}$ subband calculated from Eq. (2). The solid lines are a fit to the data points using (3) and $x=0.006$ at $90 \mathrm{~K}$ and $x=0.13$ at $190 \mathrm{~K}$.

level in the InAs quantum well. Using (1) we can write the following expression:

$$
I_{\text {leak }}^{\mathrm{L}}(p) \propto \exp \left(-\left(\frac{\mathrm{d} E_{\mathrm{L}}}{\mathrm{d} p}-\frac{\mathrm{d} E_{\Gamma}}{\mathrm{d} p}\right) \frac{p}{k T}\right),
$$

where $p$ is pressure value, $\mathrm{d} E_{\mathrm{L}} / \mathrm{d} p$ and $\mathrm{d} E_{\Gamma} / \mathrm{d} p$ are pressure coefficients of the direct $\Gamma$ and indirect $\mathrm{L}$ band gaps in the InAs quantum wells. We assume here that $\mathrm{d} E_{\mathrm{a}}=\mathrm{d} E_{\mathrm{L}}-\mathrm{d} E_{\Gamma}$.

Taking into account the pressure dependence of the $\Gamma$ and L minima (with increasing pressure the energy separation between the $\Gamma$ and $\mathrm{L}$ minima decreases at a rate of $\sim 7 \mathrm{meV} / \mathrm{kbar}$ ) [7] and using (2) we can estimate the pressure dependence of $\Gamma-\mathrm{L}$ carrier leakage.

One can see from Fig. 3 that for the $3.3 \mu \mathrm{m}$ QC laser, $I_{\text {th }}$ is almost pressure independent, which shows that carrier leakage into the L-valleys is negligible in this device even at RT. The threshold current in this case is due to phonon scattering intersubband relaxation with very weak pressure dependence due to a small pressure dependence of the phonon energy. The pressure dependence of intersubband carrier relaxation via longitudinal polar optical phonon scattering calculated for the $2.9 \mu \mathrm{m}$ device is shown in Fig. 3. However, in contrast, the measured threshold current of this device increases considerably with pressure indicating significant leakage into the L-valley. This effect becomes more pronounced with increasing temperature (see results for $90 \mathrm{~K}$ and $190 \mathrm{~K}$ in Fig. 3) in accordance with (1). The effect of pressure is approximately the same at $3.8 \mathrm{kbar}, 90 \mathrm{~K}$ and at only $2.1 \mathrm{kbar}$ at $190 \mathrm{~K}$. It was not possible to achieve lasing above approximately $2.4 \mathrm{kbar}$ and $4.2 \mathrm{kbar}$ at $190 \mathrm{~K}$ and $90 \mathrm{~K}$, respectively.
To quantify the value of the carrier leakage in $2.9 \mu \mathrm{m}$ QCLs we fit experimental points in Fig. 3 taking into account that:

$$
\frac{I_{\text {th }}}{I_{0}}=(1-x) \cdot \frac{I_{\mathrm{ph}}}{I_{\mathrm{ph} 0}}+x \cdot \frac{I_{\text {leak }}^{\mathrm{L}}}{I_{\text {leak } 0}^{\mathrm{L}}},
$$

where $I_{0}, I_{\text {pho }}$, and $I_{\text {leak } 0}^{\mathrm{L}}$ are the threshold current, phonon scattering current and leakage current at atmospheric pressure, respectively. $x$ is a fraction of $I_{0}$ due to leakage into the L-minima at atmospheric pressure. The solid lines in Fig. 3 are the fitting curves for the data at $90 \mathrm{~K}$ and $190 \mathrm{~K}$. They fit well the experimental results with $x$ value of 0.006 and 0.130 showing that the loss of current due to L-leakage increasing from $0.6 \%$ of $I_{\text {th }}$ at $90 \mathrm{~K}$ up to $13 \%$ of $I_{\text {th }}$ at $190 \mathrm{~K}$. By applying pressure we increase the leakage current up to $29 \%$ of $I_{\text {th }}$ at $2.4 \mathrm{kbar}$ at $190 \mathrm{~K}$ and up to $20 \%$ of $I_{\text {th }}$ at $4.2 \mathrm{kbar}$ at $90 \mathrm{~K}$. Using absolute values of the leakage current determined for the $2.9 \mu \mathrm{m}$ device from the fit in Fig. 3 at $90 \mathrm{~K}$ and $190 \mathrm{~K}$, together with Eq. (1) we estimate that $E_{\mathrm{a}} \approx 48 \mathrm{meV}$, which is in reasonable agreement with the expected value [3].

Figure 4 shows light-current characteristics of the $2.9 \mu \mathrm{m}$ laser measured as a function of pressure at $90 \mathrm{~K}$. One can see that the increase in the threshold current with pressure is accompanied by a decrease in the current corresponding to the maximum of the light intensity (maximum available current, $\left.I_{\max }\right)$. The characteristics of this laser at $190 \mathrm{~K}$ were similar but the effect of the pressure on $I_{\text {th }}$ was stronger than on $I_{\max }$. For a given design the maximum available current in a QCL is determined by the total rate of supply of electrons in the structure, i.e. by the injector doping level. After alignment of the structure above the turn-on voltage, the current grows quickly with applied voltage because of the fast supply of electrons from the doped injector. When the supply of electrons from the injector becomes limited at $I_{\max }$, the small increase in current

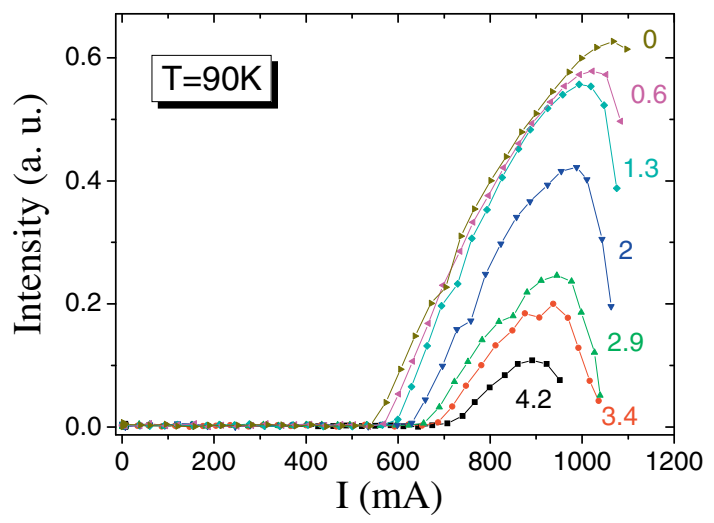

Figure 4 (online colour at: www.pss-b.com) Light-current characteristics of the $2.9 \mu \mathrm{m}$ laser measured at different pressures at $90 \mathrm{~K}$. The numbers near each curve show the pressure value in kbar. NB: laser light was measured without collecting optics. Therefore the collection efficiency may vary with pressure. This does not affect our interpretation of the results. 


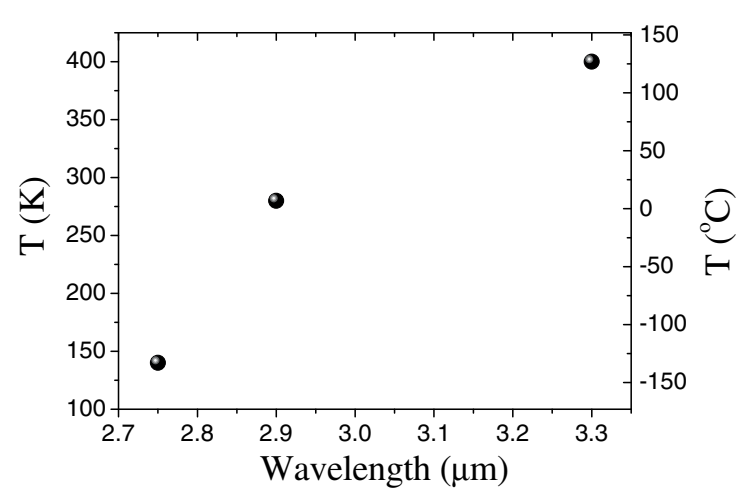

Figure $\mathbf{5}$ Maximum operating temperature as a function of wavelength for InAs/AlSb QCLs [3].

requires high growth of the electric field, which results in misalignment of the structure and ceasing of the laser action. The threshold current of the laser grows with pressure because of the reduction of population of the upper level of the lasing transition due to the leakage into L-valley. As the electron lifetime in the L-valley associated states is expected to be longer than in the $\Gamma$-levels due to the fact that the probability of indirect phonon assisted transitions is smaller than the probability of direct transitions via $\Gamma$-states, the maximum available current decreases for the same total number of electrons in the structure.

Figure 5 shows maximum operation temperature of short wavelength InAs/AlSb QCLs as a function of emission wavelength [3]. These data were obtained on longer lasers, which is responsible for their higher operation temperatures compared with results of this work. We showed that operation of the $3.3 \mu \mathrm{m}$ QCLs is not affected by the electron leakage into L-valley. It is possible to estimate now the influence of this loss on high temperature performances of the $2.9 \mu \mathrm{m}$ lasers. We used Eq. (1) and the $E_{\mathrm{a}}$ value determined above to quantify the loss due to $\Gamma-\mathrm{L}$ carrier leakage at the maximum temperature of $280 \mathrm{~K}$ as presented in [3]. Assuming that at $190 \mathrm{~K}$ the leakage current accounts up to $13 \%$ of $I_{\text {th }}$ we found that at the maximum temperature the leakage current is about $20 \%$ of $I_{\text {th }}$. This relatively small contribution of the leakage to the total current suggests that the temperature performance of such QCLs can be improved through optimisation of the design to increase the relative influence of $I_{\mathrm{ph}}$. For the QCLs emitting at $2.75 \mu \mathrm{m}$, the L-valley impact is certainly stronger in these devices and should be considered as an important factor limiting their performance.

4 Conclusion To summarise, the impact of $\Gamma-\mathrm{L}$ scattering on the temperature performance of short wavelength InAs/AlSb QCLs was investigated using high hydrostatic pressure. The results show clear evidence of the presence of this loss mechanism in lasers with emission wavelength near $\sim 2.9 \mu \mathrm{m}$, whereas it is much less pronounced in the longer wavelength $\sim 3.3 \mu \mathrm{m}$ devices allowing their superior temperature performance. We suggest that increased leakage into the L-minima is the reason for the even lower operating temperature limit $(140 \mathrm{~K})$ of lasers emitting near $2.75 \mu \mathrm{m}[3]$. In order to further reduce the operating wavelength of InAs/AlSb QCLs, carrier leakage into the indirect L-valley should be minimised to achieve laser operation over an extended temperature range.

\section{References}

[1] Y. Bai, S. R. Darvish, S. Slivken, W. Zhang, A. Evans, J. Nguyen, and M. Razeghi, Appl. Phys. Lett. 92, 101105 (2008).

[2] A. Lyakh, C. Pflügl, L. Diehl, Q. J. Wang, F. Capasso, X. J. Wang, J. Y. Fan, T. Tanbun-Ek, R. Maulini, A. Tsekoun, R. Go, C. Kumar, and N. Patel, Appl. Phys. Lett. 92, 111110 (2008).

[3] J. Devenson, O. Cathabard, R. Teissier, and A. N. Baranov, Appl. Phys. Lett. 91, 251102 (2007).

[4] J. Nguyen, Y. Bai, and M. Razeghi, Appl. Phys. Lett. 91, 141106 (2007).

[5] S. R. Jin, C. N. Ahmad, S. J. Sweeney, A. R. Adams, B. N. Murdin, H. Page, X. Marcadet, C. Sirtori, and S. Tomić, Appl. Phys. Lett. 89, 221105 (2006).

[6] S. J. Sweeney, G. Knowles, T. E. Sale, and A. R. Adams, Phys. Status Solidi B 223, 567 (2001).

[7] E. H. Li, Physica E 5, 215 (2000).

A. L. Edwards and H. G. Drickamer, Phys. Rev. 122, 1149 (1961). 